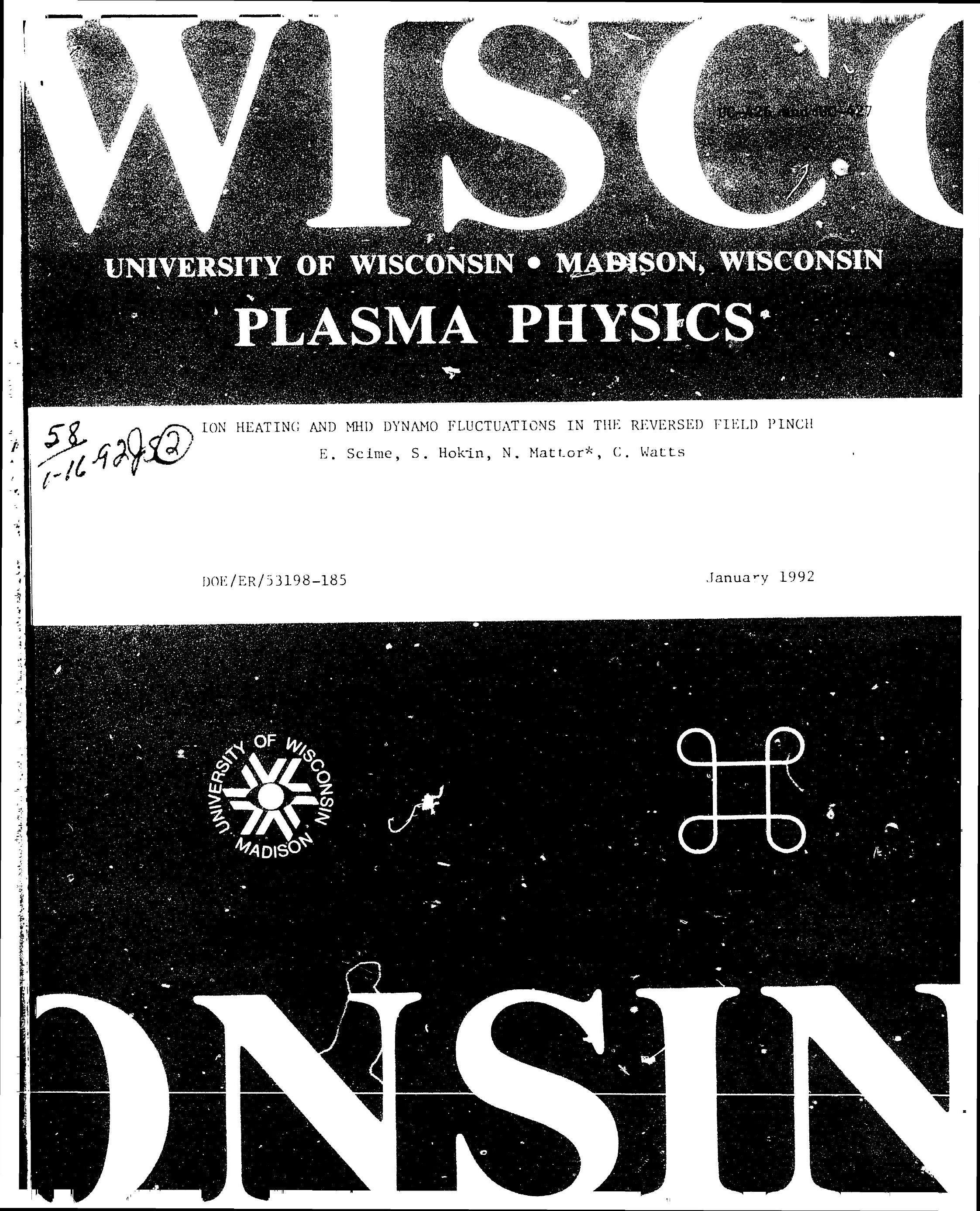




\section{NOTICE}

Tinis report was prepared as an account of work spozsored by an agency of the United States Government. Neither the United States nor any astacy thercof, nor any of their employees, makes any warranty, expressed or implied, or assumes any logal liability or responsibility for any L::-2d fusty's use or the results of luch usce of anj infornation, apparatus, product or process disclosed in this report, or represents that its use by such third party would not infringe privately owned rights.

Printed in the United States of America Available from

National Technical Information Service J.S. Department of Comnerce

5285 Port Royal Road

Springfield, VA 22161

N'TIS Price codes

Printed copy: A02

Microfiche copy: A01 


\title{
Ion Heating and MHD Dynamo Fluctuations in the Reversed Field Pinch
}

\author{
Earl Scime, Samuel Hokin, Nathan Mattor*,Christopher Watts \\ University of Wisconsin, Madison, WI 53706 \\ *present address \\ Lawrence Livermore National Laboratory, Livermore CA 94553
}

$\mathrm{DOE} / \mathrm{ER} / 53198--185$

DE92 005229

Ion temperature measurements, time resolved to $10 \mu \mathrm{s}$, have been made in the Madison Symmetric Torus reversed-field pinch with a five channel charge exchange analyzer. The ion temperature, $T_{i} \approx 200 \mathrm{eV}$ for $\mathrm{I}=350 \mathrm{kA}$, increases by as much as 100\% during discrete dynamo bursts in MST discharges. Magnetic field fluctuations in the range $0.5-5 \mathrm{MHz}$ were also measured. Structure in the fluctuation frequency spectrum at the ion cyclotron trequency appears as the bursts terminate, suggesting that the mechanism of ion heating involves the dissipation of dynamo fluctuations at ion gyro-orbit scales. 
Reveised-field pinch (RFP) experiments with ion temperature diagnostics have reported ion temperatures clearly exceeding that which would be expected from simple collisional equilibration with the Ohmically heated electrons [1-4], and some authors have suggested the dissipation of MHD dynamo fluctuations as a mechanism for energy transfer to the ions [36]. The MHD dynamo [7-9] model for RFP field sustainment requires a substantial fluctuation level to generate the reversed toroidal flux. These fluctuations should be strongly coupled to the plasma via the small resistivity. Ion inertial effects and viscosity can then convert the flow fluctuations into ion thermal energy. Arguments to support this hypothesis have typically focused upon the amount of Ohmic power that does not appear in electron heating [2,4-5], which is assumed to be stored in the fluctuations. Magnetic field fluctuations as large as a few percent are present in RFP's and previous results have shown a clear dependence of the ion temperature on the fraction of non-Ohmic power [2,4-5]. Recently there have been some attempts to correlate ion heating and soft X-ray fluctuations in REPUTE-1 [4]; however, no direct measurements of dynamo fluctuations have been available.

As reported elsewhere [10], the toroidal flux in the MST reversed-field pinch is generated in both a continuous fashion and during discrete events (Fig. 1). The self-generation of magnetic field in the RFP is attributed to an internal dynamo mechanism; therefore, the observed discrete flux generating events in MST can be interpreted as periods of increased dynamo activity. The generation of the equilibrium toroidal field has been determined to result from the nonlinear coupling of $m=1$ tearing modes (the "MHD dynamo") [10]. The magnetic field mode structure during the discrete dynamo 
bursts changes dramatically, with energy flowing rapidly from small wavenumbers to the largest wavenumbers resolvable [10]. These dynamo events in the MST discharges present an unparalleled opportunity for a careful examination of the ion heating question, and in this Letter we report the first measurements of ion temperature and magnetic field fluctuations during discrete dynamo events.

The effective ion temperature was measured using a newly developed scanning, charge exchange analyzer (CXA) (Fig. 2). The analyzer uses five continuous electron multipliers and an electrostatic bending field for energy analysis. Background plasma light was kept to a minimum and the background ion signal was eliminated by using a high voltage deflecting system upstream from the nitrogen stripping chamber. The detectors were used in a current, rather than pulse counting, mode. The time resolution of the analyzer was limited by available neutral flux to $10 \mu \mathrm{s}$. The measured signal to noise ratio of each channel exceeded 20:1 for discharges reported in this Letter, and the typical energy range analyzed was $200 \mathrm{eV}$ to $2000 \mathrm{eV}$. The ion temperatures were determined by a nonlinear fit to the five point neutral energy spectrum produced by the CXA.

On a limited number of discharges a graphite pellet was injected into the plasma. A seven-channel Doppler mear,urement of the CV $227.1 \mathrm{~nm}$ line was then possible, and the calculated Doppler ion temperature was in good agreement with the CXA hydrogen ion temperature: $\mathrm{T}_{\mathrm{i}}$ Dopp $=75 \pm 15 \mathrm{eV}$, $\mathrm{T}_{\mathrm{i}}{ }^{\mathrm{CXA}}=90 \pm 20 \mathrm{eV}$.

We find that average ion temperature during the first few milliseconds of the discharge greatly exceeds that which would be expected from simple electron-ion collisional energy transfer (for MST, $\tau_{\text {equil }} \approx 10 \mathrm{~ms}$ ) (Fig. 1). This 
result confirms the anomalous character of the ion temperature as a general feature of the RFP as reported by other groups [1-6]. We also find that the measured ion temperature increases by as much as $100 \%$ during a discrete dynamo event (Fig. 1 and Fig. 3). This result is the first published evidence that ion heating and dynamo fluctuations are correlated in the RFP.

Charge exchange measurements of the ion temperature are susceptible to changes in the neutral density and ion temperature profiles. The neutral flux simulation code NEUCGA [11-12] was used to model the neutral flux from MST, and attempts were made to reproduce the observed changes in $T_{i}$ and total neutral flux by changing the $T_{i}, T_{e}$, and $n_{e}$ profiles. The required profile changes were inconsistent with the line integrated density measurements made with a $\mathrm{CO}_{2}$ interferometer. The simulation also indicated that typical MST plasma densities are too low to affect significantly the temperature calculations based on fits to the tail of the neutral energy spectrum (Fig. 4). Therefore, the measured changes in $T_{i}$ represent actual changes in the plasma ion temperature.

Theoretical considerations [13] suggest that a turbulent MHD cascade to progressively smaller scales where $\omega \approx \omega_{\mathrm{ci}}\left(\omega_{\mathrm{ci}}\right.$ is the ion cyclotron frequency) can transfer $\tilde{B}$ energy directly to the ions. This encouraged us to investigate high frequency magnetic fluctuations during the discrete dynamo events. The fluctuations were measured at the edge of the plasma using a single electrostatically shielded coil. The signals were bandpass filtered $(0.5-5$ $\mathrm{MHz}$ ) and digitized at $10 \mathrm{MHz}$. During a dynamo burst the total power in the fluctuation signal increased by as much as three orders of magnitude (Fig. 5). After the peak generation of reversed toroidal field, a trough appears in the power spectrum at the edge ion cyclotron frequency, $f \approx f_{\mathrm{ci}} \approx 2 \mathrm{MHz}$ (Fig. 5). 
At this point, the neutral flux emission peaks, as well as the ion temperature (Fig. 3 and Fig. 4). As mentioned earlier, a cascade of energy to smaller scales during the dynamo bursts is seen in the lower frequency $(1-250 \mathrm{kHz})$ magnetic fluctuations [10].

Qualitatively, our results support the turbulent MHD cascade scenario of Ref. [13], but a more quantitative comparison is desirable. Unfortunately, single-point probes can only measure the instantaneous energy spectrum, and a quantitative comparison to the model would require measurements of the spectral enerry transfer rate. Spectral amplitude estimates from the theory of MHD inertial range turbulence (as in Ref. [13]) do not apply here, as the $\omega^{-3 / 2}$ theoretical spectrum clearly disagrees with the measured $\omega^{-3}$ spectrum (Fig. 5). It is possible, however, to compare the energy associated with anomalous loop voltage (non-Ohmic power) during the dynamo bursts given by $\int_{t_{0}}^{t} I V_{L}^{a n o m} /($ Volume $)$, with the energy gained by the ions during the same period, approximately $\frac{3}{2} n_{i} \Delta T_{i}$. For the dynamo events shown in Figure 1, the non-Ohmic power associated each burst is two to three times the energy that would be required for the observed ion temperature changes of around $100 \mathrm{eV}$. We have assumed in this calculation that the ion heating occurs at a much faster rate than ion transport; the loss of energy via ion transport during heating or partial electron heating are just two possible explanations for the discrepancy.

In summary, the measured ion temperature in the MST appears dependent upon the level of dynamo activity. The rapid ion heating and the structure of the magnetic fluctuation power spectrum during the dynamo bursts suggest energy cascading and dissipation at $\omega \approx \omega_{\mathrm{ci}}$ frequencies. T'.tese results are consistent with the hypothesis that dynamo fluctuations are 
damped at small spatial scales and are responsible for the observed ion heating in the reversed field pinch. 
1 R. B. Howell and Y. Nagayama, Phys. Fluids 28, 743 (1985).

2 P. G. Carolan, A. R. Field, A. Lazaros, M. G. Rusbridge, H. Y. W. Tsui, and M. V. Bevir, in Proceedings of the 14th European Conference on Controlled Fusion and Plasma Physics, Madrid (European Physical Society, 1987) vol. II, 469.

3 T. Fujita, K. Saito, J. Matusi, Y. Kamada, H. Morimoto, Z. Yoshida, and N. Inoue, Nucl. Fusion 31, 3 (1991).

4 A. Fujisawa, H. Ji, K. Yame gishi, S. Shinohara, H. Toyama, K. Miyamoto, Nucl. Fusion 31, 1443 (1991).

5 G. A. Wurden, P. G. Weber, K. F. Schoenberg, A. E. Schofield, J. A. Phillips, C. P. Munson, G. Miller, J. C. Ingraham, R. B. Howell, J. N. Downing, R. R. Chrien, T. E. Cayton, L. C. Burkhardt, R. J. Bastasz, S. E. Walker, A. M. Prezler, P. G. Carolan, and C. A. Bunting, in Froceedings of the 15th European Conference on Controlled Fusion and Plasma Physics, Dubrovnik (European Physical Society, 1988), 533.

6 M. Giubbilei, P. Martin, and S. Ortolani, Plasma Phys. and Cont. Fusion 32, 405 (1990).

7 E. J. Caramana, R. A. Nebel and D.D. Schnack, Phys. Fluids 26, 1305 (1983).

8 R. A. Nebel, E. J. Caramana and D.D. Schnack, Phys. Fluids B 1,1671 (1989).

9 K. Kusano and T. Sato, Nucl. Fus. 27, 821 (1987).

10 T. Rempel, et al., submitted to Phys. Fluids B.

11 K. H. Burrell, J. Comp. Physics 27, 88 (1978).

12 R. M. Mayo and L. S. Kirchenbaum, Phys. Fluids B 3, 2096 (1991).

13 N. Mattor, submitted to Phys. Rev. Lett. 


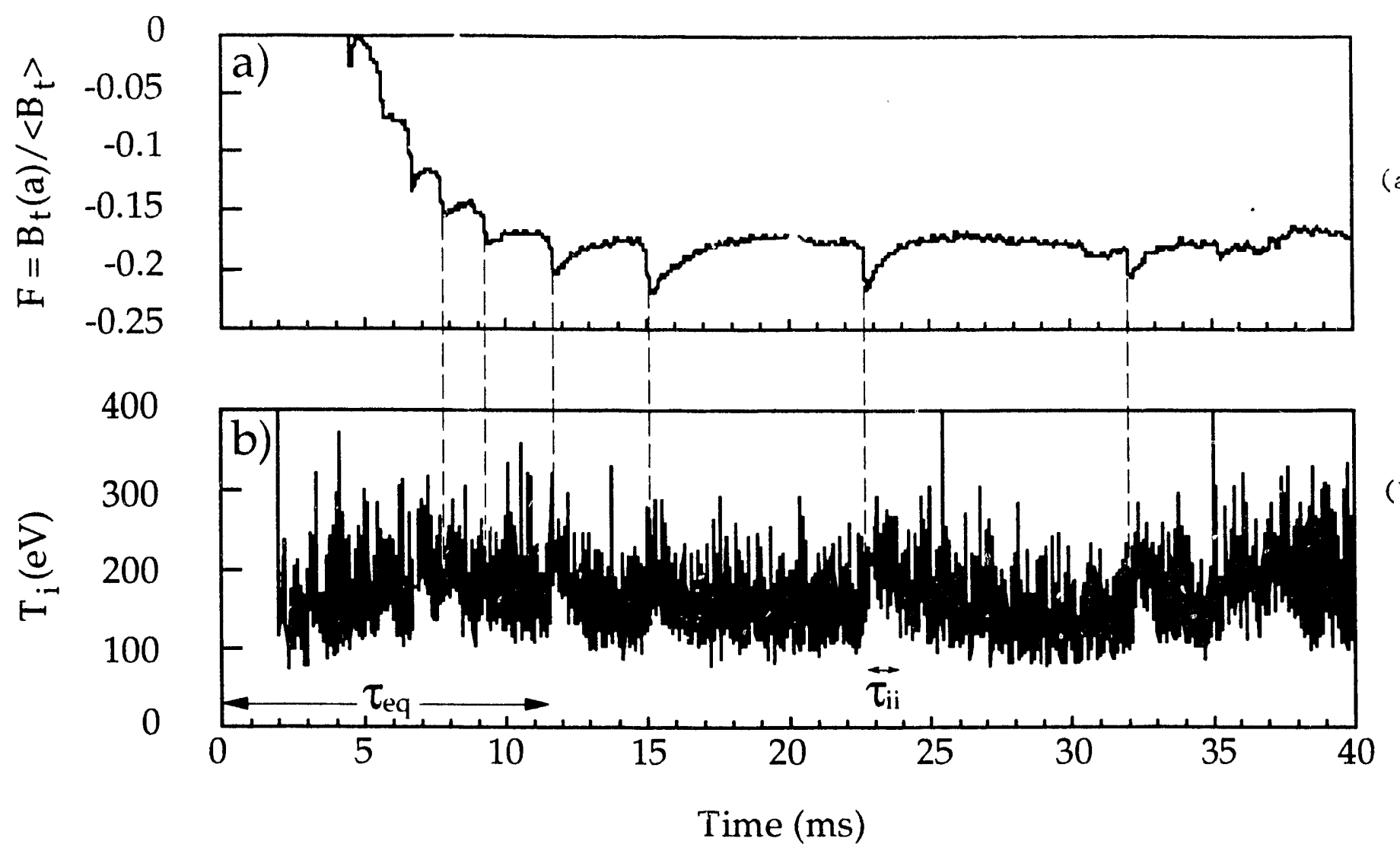

Figure 1. a) Reversed edge toroidal field is generated continuously and in discrete bursts. b) Ion temperature "bursts" correlate with dynamo activity and $\mathrm{T}_{\mathrm{i}} \approx 100 \mathrm{eV}$ within $2 \mathrm{~ms}$ of startup. 


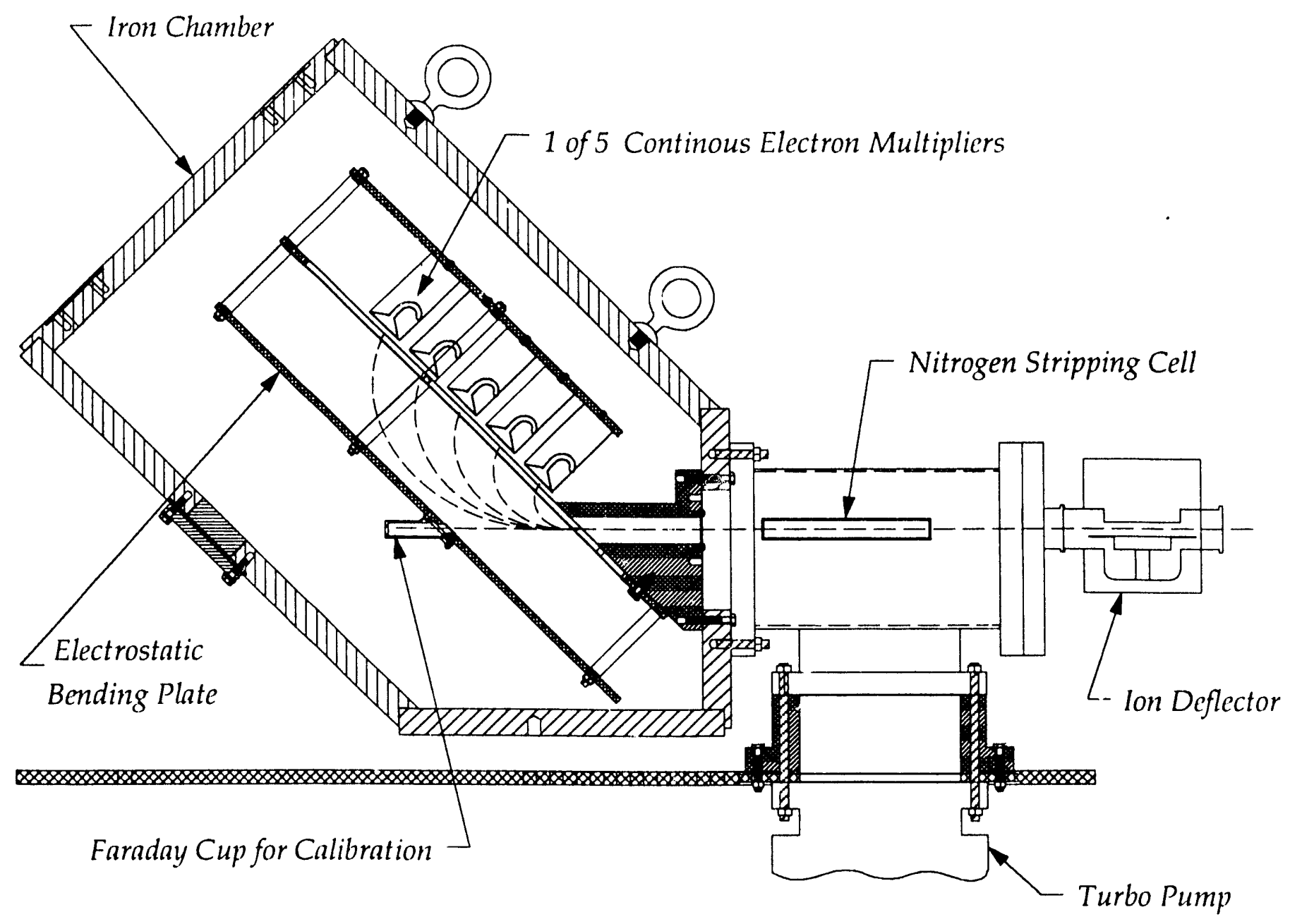

Figure 2. Schematic of the five-channel charge exchange analyzer. 


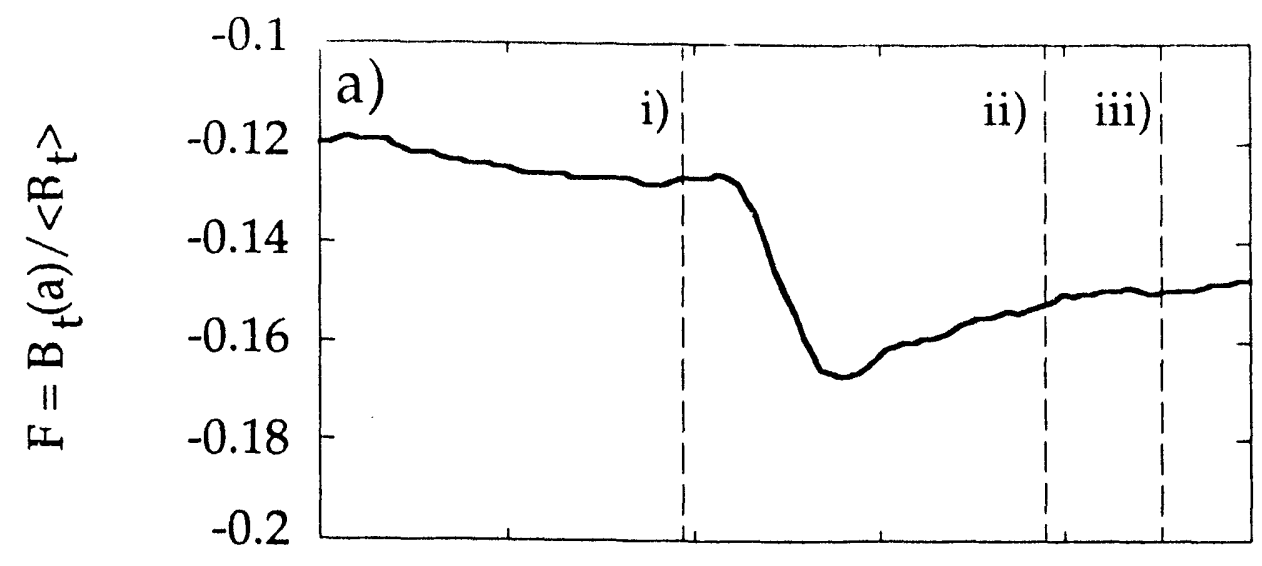

(a)

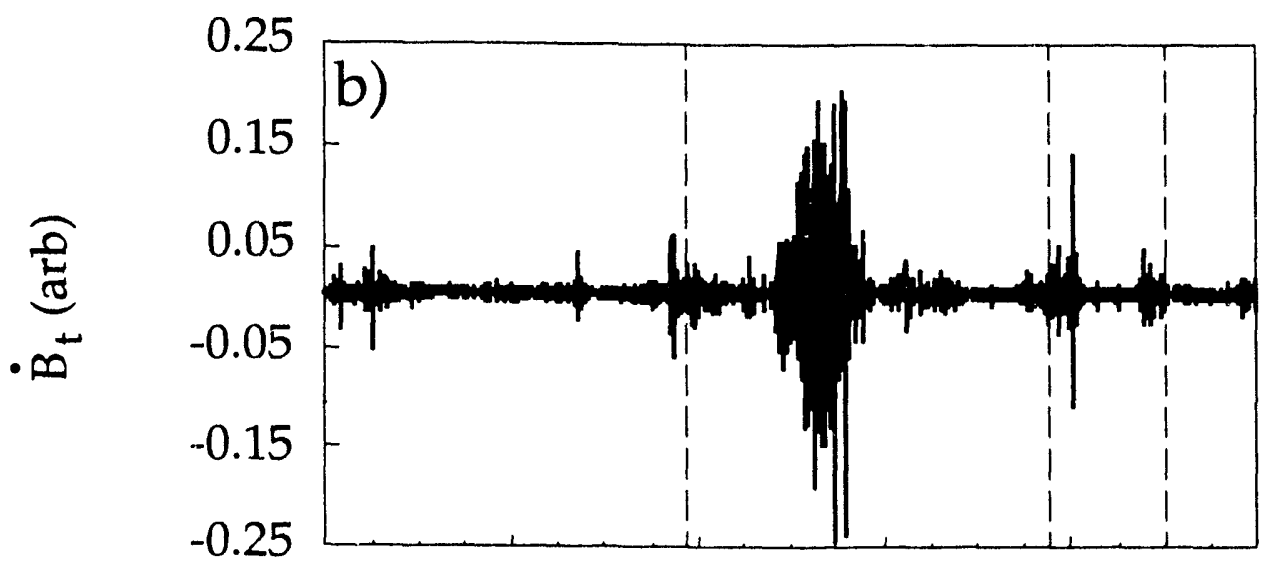

(b)

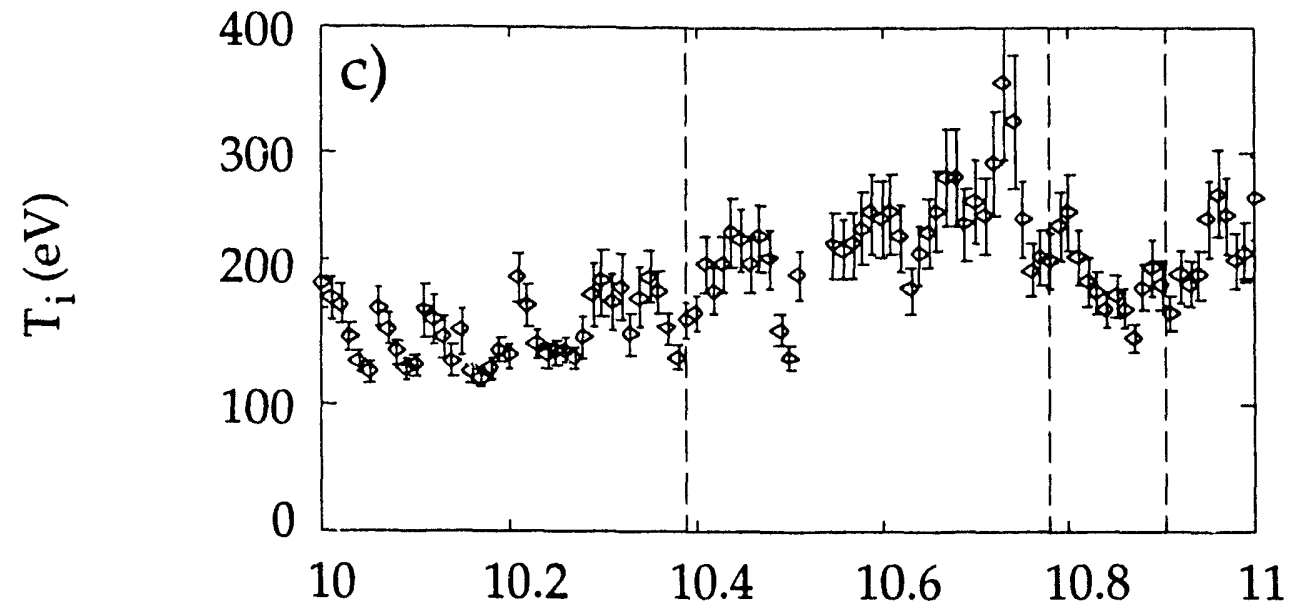

(c)

Figure 3. a) Enlarged view of reversal parameter during a dynamo burst [not the same discharge as Fig. 1]. b) High frequency, 0.5 - $5.0 \mathrm{MHz}$, toroidal magnetic field fluctuations during the same time interval.

c) Ion temperature from CXA during the same interval. 


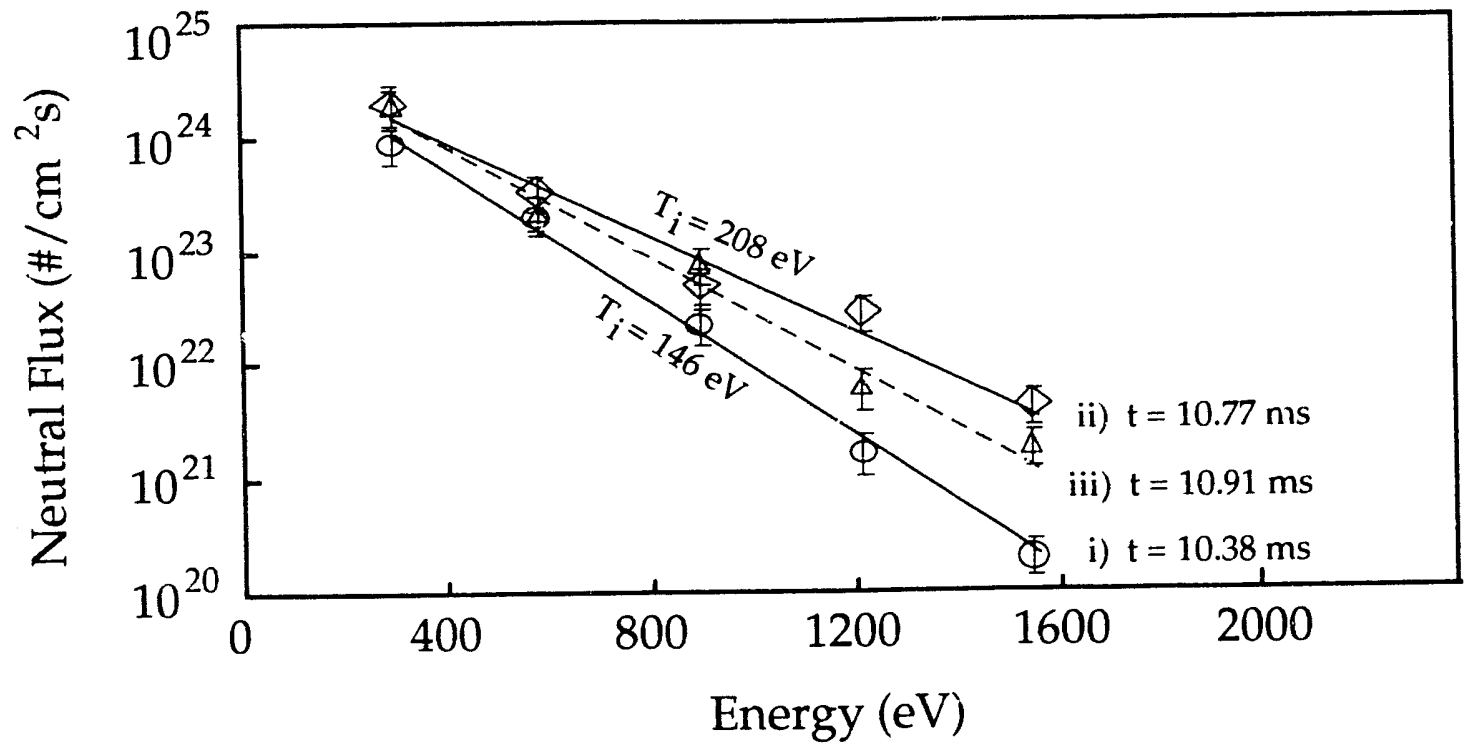

Figure 4. Neutral energy spectra during a dynamo burst. Times indicated by lower-case roman numerals correspond to those in Fig. 3. 


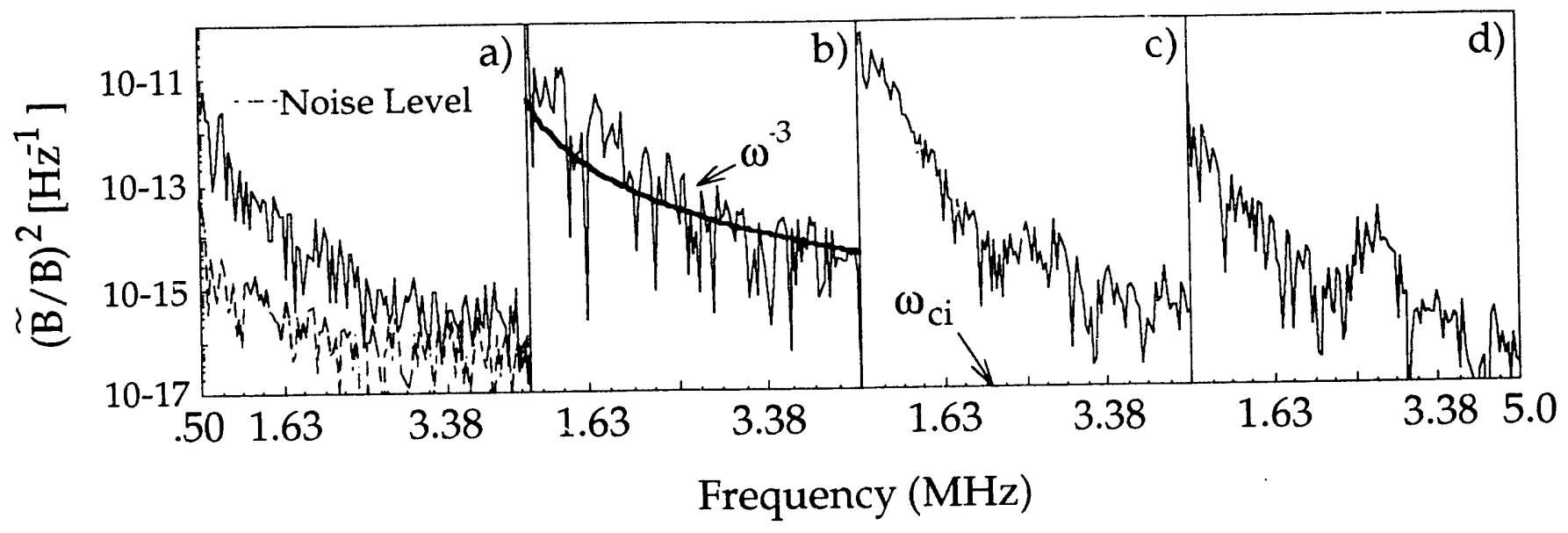

Figure 5. Typical magnetic power spectra a) before, b-c) during, and d) after a dynamo burst; each interval corresponds to $25 \mathrm{~ms}$. 


\section{EXTERNAL DISTRIBUTION IN ADDITION TO UC-20}

S.N. Rasband, Brigham Young University

J.B. Taylor, Institute for Fusion Studies, The University of Texas at Austin M.A. Abdou, University of California, Los Angeles

R.W. Conn, University of California, Los Angeles

T. Dolan, INEL

R. Smith, University of lowa

F.W. Perkins, PPPL

O. Ishihara, Texas Technical University

P.E. Vandenplas, Association Euratom-Etat Belge, Belgium

Centro Brasileiro de Pesquisas Firicas, Brazil

P. Sakanaka, Institute de Fisica-Unicamp, Brazil

Mme. Monique Bex, GANIL, France

J. Radet, CEN/CADARACHE, France

University of loannina, Greece

S. Ortolani, Istituto Gas Ionizzati, EURATON-ENEA-CNR Association, Italy

R. Andreani, Associazione EURATOM-ENEA sulla Fusione, Italy

Y. Kondoh, Gunma University, Kiryu, Gunma, Japan

H. Toyama, University of Tokyo, japan

FOM-Instituut voor Plasmafysica "Rijnhuizen", The Netherlands

Z. Ning, Academia Sinica, People's Republic of China

P. Yang, Shandong University, People's Republic of China

S. Zhu, University of Science \& Technology of China, People's Republic of China

I.N. Bogatu, lıstitute of Atomic Physics, Romania

M.J. Alport, University of Natal, Durban, South Africa

R. Storer, The Flinders University of South Australia, South Australia

B. Lehnert, Royal Institute of Technology, Sweden

Librarian, CRPP, Ecole Polytechnique Federale de Lausanne, Switzerland

B. Alper, Culham Laboratory, UK

A. Newton, Culham Laboratory, UK

6 for Chicago Operations Office

4 for individuals in Washington Offices

INTERNAL DISTRIBUTION IN ADDITION TO UC-20

80 for local group and file 

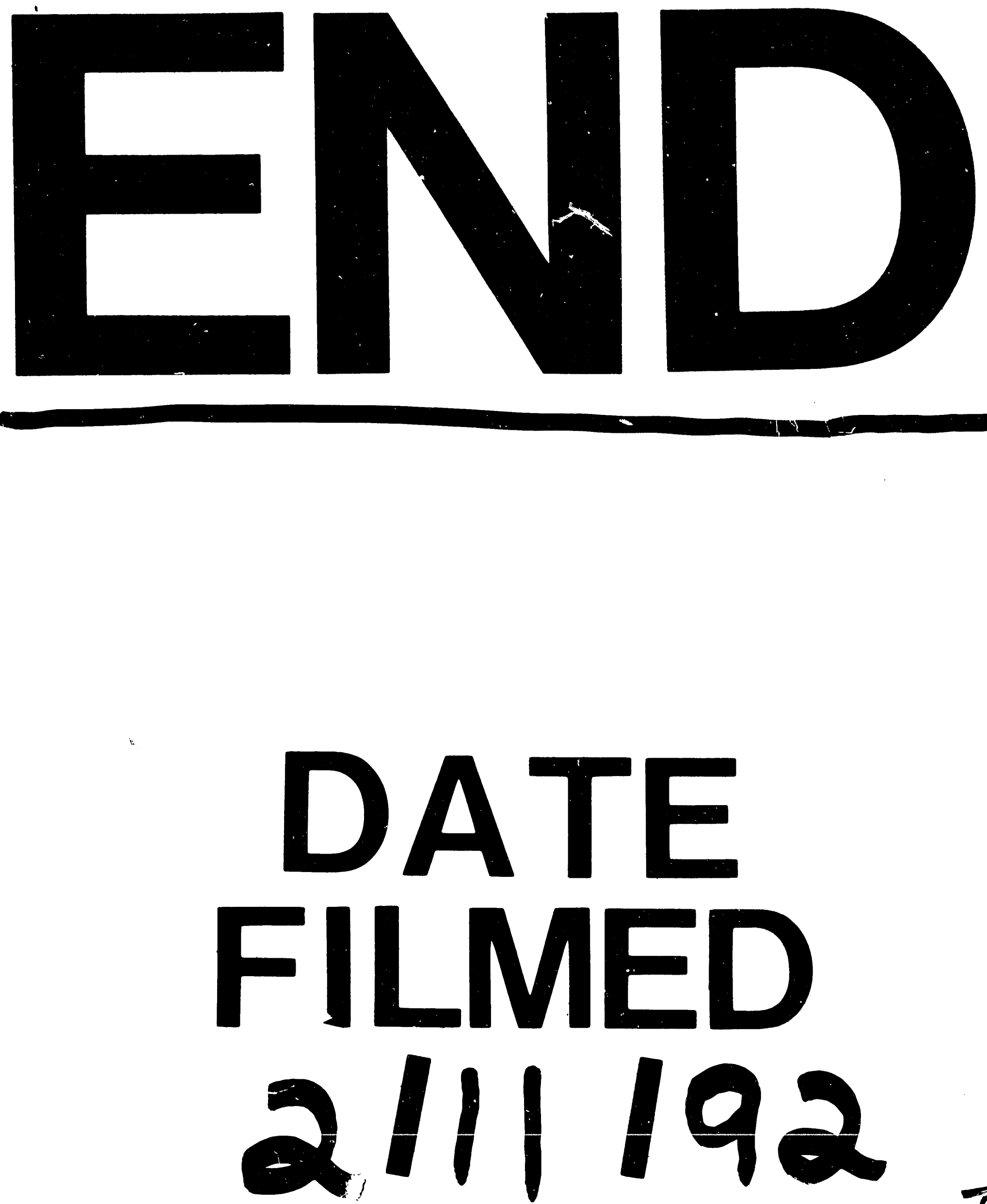

I 


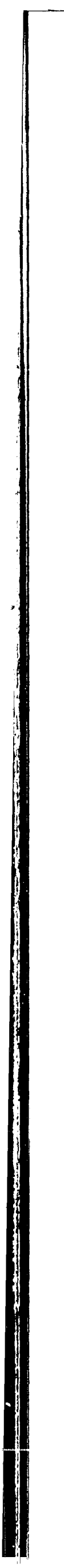

